\title{
Aducanumab produced a clinically meaningful benefit in association with amyloid lowering
}

\author{
Jeffrey Cummings ${ }^{1 *}$ (D), Paul Aisen ${ }^{2}$, Cynthia Lemere ${ }^{3}$, Alireza Atri ${ }^{4,5}$, Marwan Sabbagh $^{6}$ and Stephen Salloway ${ }^{7}$
}

Keywords: Aducanumab, FDA, Clinical trials, Monoclonal antibodies, Lecanemab, Donanemab, Gantenerumab

Aducanumab is a monoclonal antibody targeting amyloid beta protein $(A ß)$, a defining feature of the biology of Alzheimer's disease (AD) [1]. Laboratory studies showed high affinity of aducanumab for the neurotoxic oligomeric species of $A ß$ [2]. Following a promising phase 1B trial [3], the sponsor (Biogen) implemented two phase 3 studies-EMERGE and ENGAGE. A planned futility analysis concluded that the treatment was not beneficial, and the trials were terminated. With the accrual of additional blinded data, the prespecified analysis of the primary outcome-Clinical Dementia Rating Sum of Boxes (CDR-sb)-showed that the EMERGE trial met its primary outcome and the ENGAGE trial did not. Biogen submitted the data to the US Food and Drug Administration (FDA) for review and possible marketing approval, setting the stage for a vigorous dialogue on aducanumab $[4,5]$.

The CDR-sb, comprising the primary outcome of ENGAGE and EMERGE, is a composite measure with cognitive and functional components including home activities, problem solving, and community engagement-skills highly valued by patients [6]. In EMERGE, aducanumab treatment resulted in a significant $22 \%$ slowing of decline on the CDR-sb [7]. This instrument has a restricted range (0-18); small changes reflect meaningful clinical alterations. In both trials, participants who received at least 14

\footnotetext{
* Correspondence: jcummings@cnsinnovations.com

${ }^{1}$ Chambers-Grundy Center for Transformative Neuroscience, Department of Brain Health, School of Integrated Health Sciences, University of Nevada Las Vegas (UNLV), Las Vegas, NV, USA

Full list of author information is available at the end of the article
}

doses of the highest dose of aducanumab showed similar levels of slowing on the CDR-sb (30\% in EMERGE, 27\% in ENGAGE). In EMERGE, all secondary measures including the Mini Mental State Examination, Alzheimer's Disease Assessment Scale-cognitive subscale, and the Alzheimer's Disease Cooperative Study Activities of Daily Living (ADCS ADL) scale showed statistically significant drugplacebo differences. The ADCS-ADL scale showed a robust $40 \%$ slowing of functional decline in the treatment group compared to the placebo group [7]. The Neuropsychiatric Inventory (NPI) that assesses an array of behavioral changes common in AD showed an 87\% reduction from baseline scores in the high dose group of EMERGE [8]. There was a corresponding $84 \%$ reduction in caregiver distress. Disease-modifying therapies change the trajectory of disease progression; benefits observed in trials are anticipated to increase with long-term treatment. Extending the mild cognitive impairment stage of $\mathrm{AD}$ and delaying the dementia stage is very meaningful for a 68 year-old grandmother seeking to preserve daily activities, hobbies, and community and family engagement.

Amyloid plaques measured by amyloid positron emission tomography (PET) were markedly decreased by aducanumab in both trials. Phosphorylated tau (p-tau) in the cerebrospinal fluid (CSF) and medial temporal neurofibrillary tangles measured by tau PET in a small subset of patients were reduced as predicted by "the amyloid hypothesis." Phosphorylated tau is closely linked to cognitive decline [1]. Statistically significant correlations were present between $A ß$ reduction and the 
clinical outcomes of EMERGE and between $A ß$ reduction and CSF p-tau changes [8].

An argument marshaled against accepting the EMERGE trial as evidence of efficacy is that previous clinical trials of drugs targeting $A ß$ have been negative [4]. This view fails to account for recent promising clinical trials specifically involving anti-Aß monoclonal antibodies including lecanemab [9], gantenerumab [10], and donanemab [11] and the many learnings that have occurred concerning dose, targeting specific types of $A ß$, and treating patients earlier in the disease [12]. Doses of monoclonal antibodies have more than quadrupled from those used in previous trials as more evidence has informed exposure requirements, and study populations have shifted toward earlier intervention prior to extensive irreversible neurodegeneration. The recently reported positive donanemab phase 2 trial, linking reduction of brain amyloid with cognitive/functional benefit [11], provides particularly strong support for the therapeutic approach of aducanumab. In view of these recent findings, EMERGE results can be considered consistent with other similar studies rather than as an anomaly.

Aducanumab and several other monoclonal antibodies are associated with amyloid-related imaging abnormalities (ARIA) thought to represent effusion through the blood-brain barrier (ARIA-E) or hemorrhages (ARIA-H) associated with blood-brain barrier compromise. ARIAE occurred in $34 \%$ and $35.5 \%$ of those receiving highdose aducanumab in EMERGE and ENGAGE respectively. Most (80\%) ARIA events are without symptoms. When symptoms occur, they include headache, dizziness, visual disturbances, and nausea. ARIA is a manageable side effect of treatment with aducanumab and far less compromising than complications of many routinely used cancer therapies.

Although not directly relevant to determining the efficacy and safety of aducanumab, criticism has been directed at the FDA for working too closely with Biogen in the submission process [13]. FDA has provided written guidance for regularly scheduled meetings with all sponsors and works closely with sponsors to ensure clear communication regarding trial expectations and outcome interpretations [14]. The FDA decision regarding aducanumab carries great significance for patients with $\mathrm{AD}$ and their families; close communication is required to reassure those whose lives could be altered by such a therapy that all due considerations have been observed.

In the EMERGE trial, aducanumab met its primary outcome and had beneficial effects on cognition, function, and behavior. Benefits were observed in ENGAGE participants who were treated with the high dose for longer periods. A decision not to approve aducanumab in spite of these outcomes will adversely affect the field of $\mathrm{AD}$ treatment research, discouraging biopharmaceutical companies from investing in this area [15]. The first treatment of $\mathrm{AD}$, tacrine, had flaws, but it was a breakthrough that demonstrated the possibility of improving cognition in $\mathrm{AD}$ and was soon followed by improved and now widely used agents. We anticipate a similar reinvigoration of $\mathrm{AD}$ treatment research if aducanumab becomes publicly available.

An FDA Advisory Committee voted to recommend that the Agency not approve aducanumab based on a single positive study [4]. We believe that the perspective of the panel was too narrow, ignoring important scientific and clinically meaningful considerations. Based on the review of the totality of the data and our extensive experience with $\mathrm{AD}$ trials, research, and clinical care of patients and families, we conclude that aducanumab achieves the standard of meaningful efficacy with adequate safety in early $\mathrm{AD}$. We support providing persons with $\mathrm{AD}$, who face a progressive and incurable disease, with the option of making informed choices about their health and lives with respect to a first-generation drug with aducanumab's risk-burden/benefit profile.

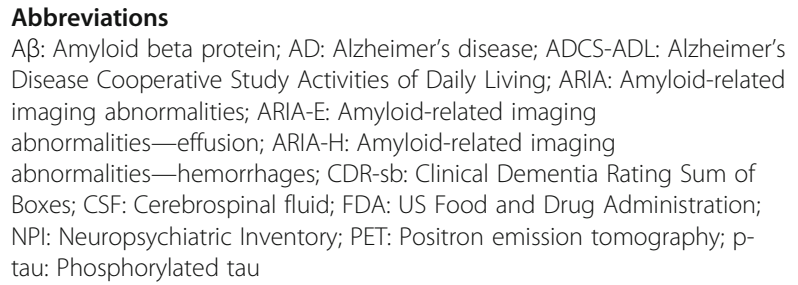

\section{Acknowledgements}

JC and MS have the following research support: NIGMS P20GM109025 and NIA P20AG068053; JC has the following research support: NINDS

U01NS093334; NIA R01AG053798 and R35AG71476.

\section{Authors' contributions}

All authors participated in the conceptualization of this paper, participated in its writing, and approved the final manuscript.

\section{Authors' information}

Not applicable.

Funding

Not applicable.

Availability of data and materials

Not applicable.

\section{Declarations}

Ethics approval and consent to participate Not applicable.

\section{Consent for publication}

Not applicable.

\section{Competing interests}

PA has received research funding from NIA, FNIH, the Alzheimer's Association, Janssen, Lilly, and Eisai and personal fees from Biogen, Merck, Roche, Abbvie, ImmunoBrain Checkpoint, Rainbow Medical, and Shionogi. CL has provided consultation to Acumen Pharmaceutical, Apellis

Pharmaceutical, Biogen (ADvance Medical Education International Working Group), Cambridge Healthcare Research, and Cognition Therapeutics. She received in-kind research support from Vivoryon Therapeutics. JC has 
provided consultation to Acadia, Alkahest, AriBio, Avanir, Axsome, Behren Therapeutics, Biogen, Cassava, Cerecin, Cerevel, Cortexyme, EIP Pharma, Eisai, GemVax, Genentech, Green Valley, Grifols, Janssen, Jazz, Karuna, Merck, Novo Nordisk, Otsuka, ReMYND, Resverlogix, Roche, Signant Health, Sunovion, Suven, United Neuroscience, and Unlearn Al pharmaceutical and assessment companies. Dr. Cummings has stock options in ADAMAS, AnnovisBio, MedAvante, BiOasis, and United Neuroscience. Dr. Cummings owns the copyright of the Neuropsychiatric Inventory. Dr Cummings has the following research support: NIGMS P20GM109025; NINDS U01NS093334; NIA R01AG053798; NIA P20AG068053; NIA R35AG71476. AA has received honoraria for consulting; participating in independent data safety monitoring boards; providing educational lectures, programs, and materials; or serving on advisory boards for AbbVie, Acadia, Allergan, the Alzheimer's Association, Axovant, AZ Therapies, Biogen, Grifols, Harvard Medical School Graduate Continuing Education, JOMDD, Lundbeck, Merck, Roche/Genentech, Novo Nordisk, Sunovion, and Suven. MS has provided consultation to Alzheon, Biogen, Cortexyme, RocheGenentech, Stage 2 Innovations/Renew Research, Acadia, T3D, Eisai, and KeifeRx. He is on the Speaker's Bureau for Health and Wellness Partners. He holds stock/options in rain Health Inc, NeuroTau, Optimal Cognitive Health Company, uMethod Health, Versanum, Athira, and Cognoptix. SS was a site $\mathrm{PI}$ and co-chair of the investigator steering committee for the ENGAGE trial, and he receives research support and consultancy fees from Lilly, Biogen, Avid, Eisai, Genentech, and Roche.

\section{Author details}

${ }^{1}$ Chambers-Grundy Center for Transformative Neuroscience, Department of Brain Health, School of Integrated Health Sciences, University of Nevada Las Vegas (UNLV), Las Vegas, NV, USA. ² University of Southern California, San Diego, CA, USA. ${ }^{3}$ Ann Romney Center for Neurologic Diseases, Brigham and Women's Hospital, Harvard Medical School, Boston, MA, USA. ${ }^{4}$ Banner Sun Health Research Institute, Banner Health, Sun City, AZ, USA. ${ }^{5}$ Center for Brain/ Mind Medicine, Department of Neurology, Brigham and Women's Hospital, Harvard Medical School, Boston, MA, USA. ${ }^{6}$ Cleveland Clinic Lou Ruvo Center for Brain Health, Las Vegas, NV, USA. ${ }^{7}$ Butler Hospital and Brown University, Providence, RI, USA.

Received: 26 April 2021 Accepted: 29 April 2021

Published online: 10 May 2021

\section{References}

1. Jack CR Jr, Bennett DA, Blennow K, Carrillo MC, Dunn B, Haeberlein SB, et al. NIA-AA Research Framework: toward a biological definition of Alzheimer's disease. Alzheimers Dement. 2018;14(4):535-62. https://doi.org/10.1016/j.ja lz.2018.02.018

2. Arndt JW, Qian F, Smith BA, Quan C, Kilambi KP, Bush MW, et al. Structural and kinetic basis for the selectivity of aducanumab for aggregated forms of amyloid-beta. Sci Rep. 2018;8(1):6412. https://doi.org/10.1038/s41598-018-24 501-0.

3. Sevigny J, Chiao P, Bussiere T, Weinreb PH, Williams L, Maier M, et al. The antibody aducanumab reduces Abeta plaques in Alzheimer's disease. Nature. 2016;537(7618):50-6. https://doi.org/10.1038/nature19323.

4. Alexander GC, Emerson S, Kesselheim AS. Evaluation of aducanumab for Alzheimer disease: scientific evidence and regulatory review involving efficacy, safety, and futility. JAMA. 2021. https://doi.org/10.1001/jama.2021.3 854.

5. Sabbagh MN, Cummings J. Open Peer Commentary to "Failure to demonstrate efficacy of aducanumab: an analysis of the EMERGE and ENGAGE Trials as reported by Biogen December 2019". Alzheimers Dement. 2021;17(4):702-3. https://doi.org/10.1002/alz.12235.

6. Watson J, Saunders S, Muniz Terrera G, Ritchie C, Evans A, Luz S, et al. What matters to people with memory problems, healthy volunteers and health and social care professionals in the context of developing treatment to prevent Alzheimer's dementia? A qualitative study. Health Expect. 2019; 22(3):504-17. https://doi.org/10.1111/hex.12876.

7. Haeberlein SB, von Hehn C, Tian Y, et al., editors. EMERGE and ENGAGE topline results: two phase 3 studies to evaluate aducanumab in patients with early Alzheimer's disease. San Diego, CA: 12th Clinical Trials on Alzheimer's Disease; 2019.

8. Haeberlein SB. Aducanumab for the treatment of Alzheimer's disease: Presentation. US Food and Drug Administration. Peripheral and Central Nervous System Drugs Advisory Board; 2020.
9. Swanson CJ, Zhang Y, Dhadda S, Wang J, Kaplow J, Lai RYK, et al. A randomized, double-blind, phase $2 \mathrm{~b}$ proof-of-concept clinical trial in early Alzheimer's disease with lecanemab, an anti-Abeta protofibril antibody. Alzheimers Res Ther. 2021;13(1):80. https://doi.org/10.1186/s13195-02100813-8.

10. Klein G, Delmar P, Voyle N, Rehal S, Hofmann C, Abi-Saab D, et al. Gantenerumab reduces amyloid-beta plaques in patients with prodromal to moderate Alzheimer's disease: a PET substudy interim analysis. Alzheimers Res Ther. 2019;11(1):101. https://doi.org/10.1186/s13195-019-0559-z.

11. Mintun MA, Lo AC, Duggan Evans C, Wessels AM, Ardayfio PA, Andersen SW, et al. Donanemab in early Alzheimer's disease. N Engl J Med. 2021.

12. Cummings J, Feldman $\mathrm{HH}$, Scheltens $P$. The "rights" of precision drug development for Alzheimer's disease. Alzheimers Res Ther. 2019;11(1):76. https://doi.org/10.1186/s13195-019-0529-5.

13. Public Citizen's Health Research Group. Request for an Office of Inspector General investigation of the Food and Drug Administration's inappropriate close collaboration with Biogen before and after the submission of the biologics license application for aducanumab for treatment of Alzheimer's disease; Public Citizen's Health Research Group; 2020 accessed 02/19/2021; Available from: https://mkus3lurbh3lbztg254fzode-wpengine.netdna-ssl. com/wp-content/uploads/2660.pdf.

14. Food and Drug Administration, Center for Drug Evaluation and Research. Formal meetings between the FDA and sponsors or applicants of PDUFA Products Guidance for Industry. Silver Spring, MD: Office of Communictions, Division of Drug Information, Center for Drug Evaluation and Research, Food and Drug Administration; 2017.

15. Editorial Board. The battle over an Alzheimer's treatment; Biogen's promising drug is caught in the FDA's political and bureaucratic limbo. Wall Street J. 2021

\section{Publisher's Note}

Springer Nature remains neutral with regard to jurisdictional claims in published maps and institutional affiliations. 